\title{
Psychosocial risk factors for postpartum depression in Chinese women: a meta- analysis
}

Weijing Qi ${ }^{\dagger}$, Fuqing Zhao ${ }^{\dagger}$, Yutong Liu, Qing Li and Jie Hu*

\begin{abstract}
Background: Postpartum depression (PPD) has been identified as a recognized public health problem that may adversely affect mothers, infants, and family units. Recent studies have identified risk factors for PPD in Westerners; however, societal and cultural differences between China and the West could, potentially, lead to differences in risk factors for PPD. No comprehensive study has been conducted to collect all the evidence to provide estimates of psychological and social risk factors in China. Therefore, this study aimed to quantitatively assess all studies meeting the review's eligibility criteria and identify the psychological and social risk factors for PPD in Chinese women.

Methods: The following databases were used in the literature search from their inception until December 2020: PubMed, Embase, Foreign Medical Literature Retrieval Service (FMRS), China Science and Technology Journal Database (VIP), China National Knowledge Infrastructure (CNKI), and China Biology Medicine disc (CBM). The quality was assessed through Newcastle-Ottawa quality assessment scale. The I'statistic was used to quantify heterogeneity. We extracted data for meta-analysis and generated pooled-effect estimates from a fixed-effects model. Pooled estimates from a random-effects model were also generated if significant heterogeneity was present. Funnel plot asymmetry tests were used to check for publication bias. Statistical analysis was conducted using Review Manager version 5.3 software.

Results: From a total of 1175 identified studies, 51 were included in the analysis. Prenatal depression (OR 7.70; 95\% $\mathrm{Cl} 6.02-9.83)$ and prenatal anxiety $(\mathrm{OR} 7.07 ; 95 \% \mathrm{Cl}$ 4.12-12.13) were major risk factors for PPD. A poor economic foundation (OR 3.67; 95\% Cl 3.07-4.37) and a poor relationship between husband and wife (OR 3.56; 95\% Cl 2.954.28) were moderate risk factors. Minor risk factors included a poor relationship between mother-in-law and daughter-in-law (OR 2.89; 95\% Cl 2.12-3.95), a lack of social support (OR 2.57; 95\% Cl 2.32-2.85), unplanned pregnancy (OR 2.55; 95\% Cl 2.08-3.14), and poor living conditions (OR 2.44; 95\% Cl 1.92-3.10), mother-in-law as the caregiver $(1.95 ; 95 \% \mathrm{Cl} 1.54-2.48)$.
\end{abstract}

Conclusions: This study demonstrated a number of psychological and social risk factors for PPD in Chinese women. The major and moderate risk factors are prenatal depression, prenatal anxiety, a poor economic foundation, and a poor relationship between husband and wife. These findings have potential implications for informing preventive efforts and modifying screening to target at-risk populations.

Keywords: Postpartum depression, Risk factors, Meta-analysis, Psychosocial

\footnotetext{
*Correspondence: hujie3993@126.com

${ }^{+}$Weijing Qi and Fuqing Zhao contributed equally to this work.

Department of Clinical Humanistic Care and Nursing Research Center, School of Nursing, Hebei Medical University, Dr. 361 East Zhongshan Road,

Shijiazhuang 050017, Hebei Province, China
}

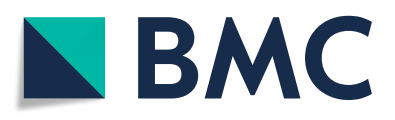

(c) The Author(s). 2021 Open Access This article is licensed under a Creative Commons Attribution 4.0 International License, which permits use, sharing, adaptation, distribution and reproduction in any medium or format, as long as you give appropriate credit to the original author(s) and the source, provide a link to the Creative Commons licence, and indicate if changes were made. The images or other third party material in this article are included in the article's Creative Commons licence, unless indicated otherwise in a credit line to the material. If material is not included in the article's Creative Commons licence and your intended use is not permitted by statutory regulation or exceeds the permitted use, you will need to obtain permission directly from the copyright holder. To view a copy of this licence, visit http://creativecommons.org/licenses/by/4.0/. The Creative Commons Public Domain Dedication waiver (http://creativecommons.org/publicdomain/zero/1.0/) applies to the data made available in this article, unless otherwise stated in a credit line to the data. 


\section{Background}

Postpartum depression (PPD) is the most common type of nonpsychotic psychiatric syndrome during the perinatal period [1]. The Diagnostic and Statistical Manual of Mental Disorders Fifth Edition (DSM-5) defines PPD as a depressive episode with moderate-to-severe symptoms that begins 4 weeks after delivery [2]. The prevalence of PPD varies from 0.5 to $60.8 \%$ around the world and from 3.5 to $63.3 \%$ in Asian countries, as measured using the Edinburgh Postpartum Depression scale (EPDS) [3]. The prevalence of PPD in China is $27.37 \%$ [4], and is increasing yearly [5]. PPD has been identified as a recognized public health problem that not only affects mothers' health but also causes poor developmental outcomes in children and poor relationships in families [6].

Postpartum depressive symptoms include the inability to sleep, anxiety, sadness, extreme concern and worry about the baby, and even recurrent thoughts of death [7]. Because maternal emotion plays an important role in the development of children, the pathogenesis of PPD merits greater attention. At present, studies involving Chinese women have reported that poor relationships with husbands or mothers-in-law, introverted maternal personality, anxiety or depression during pregnancy, an unsmooth delivery process, poor postpartum sleep quality, dissatisfaction with neonatal sex, and poor health conditions of newborns were risk factors for PPD [8]. Although several risk factors have been identified, the results of some risk factors are still controversial. Of note, these reviews included some case-control studies and cross-sectional studies, which limited the strength and quality of such evidence.

In addition, review [9] on the risk factors for PPD have primarily included studies conducted in Western populations and have overlooked many studies undertaken in the Chinese cultural context. Western and Chinese women differ considerably in terms of genetics, philosophical traditions, cultural practices, ethnicity, religion, and attitudes toward psychological problems [10]. The two different cultural backgrounds lead to differences in the psychosocial risk factors for PPD [11]. Given the deeply rooted influence of the Chinese cultural traditions of Confucianism, Buddhism and Taoism, the thought processes of Chinese females have historically been more traditional and conservative in nature [12]. Some studies have shown that culturally and characteristically Westerners are more extroverted, while characteristically Chinese people are more introverted introverted women with personality types that are dependent are more prone to experience PPD [13, 14].

In addition, although some Chinese individuals have emigrated to other countries, they have been influenced by traditional Chinese culture for a long time; thus their personality and their family structure are still similar to those of individuals in their motherland. In particular, many Chinese immigrant women continue to observe the Chinese traditional postpartum practices. Thus the aim of this review was to synthesis the evidence from eligible studies to identify the psychosocial risk factors for PPD in Chinese women, including those living in other countries.

\section{Methods}

\section{Search strategy}

A systematic search of the electronic databases PubMed, Embase, Foreign Medial Literature Retrieval Service (FMRS), the China Science and Technology Journal Database (VIP), China National Knowledge Infrastructure (CNKI), and China Biology Medicine disc (CBM) was performed for relevant studies published before June 2019. Chinese search terms included PPD, risk factors, influencing factors, social factors, and psychological factors. English search terms included the following: Postnatal Depression OR Depression, Postnatal OR Post-Partum Depression OR Depression, Post-Partum OR Post Partum Depression OR Postpartum Depression OR Post-Natal Depression OR Depression, Post-Natal OR Post Natal Depression AND Factor, Risk OR Factors, Risk OR Risk Factor OR Population at Risk OR Risk, Population at OR Populations at Risk OR Risk, Populations at AND Chinese OR in China. Additionally, we also performed a manual search of the reference lists of retrieved articles and recent reviews.

\section{Population (P)}

Chinese women who have given birth to at least one child, including those living in countries other than China.

\section{Exposure (E)}

A woman who has experienced childbirth and has been exposed to a risk factor for PPD.

\section{Outcome (0)}

PPD is the most common type of nonpsychotic psychiatric syndrome during the perinatal period, as a depressive episode with moderate-to-severe symptoms that begins 4 weeks after delivery.

\section{Selection criteria}

This systematic review and meta-analysis considered all studies on psychosocial risk factors for PPD in Chinese women, including Chinese women currently living in other countries. 


\section{Exclusion criteria}

The title and abstracts of retrieved studies were assessed against the review's inclusion criteria. Papers that were inaccessible, and those that did not report associated risk factors for PPD were excluded. Studies that were not a case-control study or a cohort study were also excluded. Once more, studies that did not report relative risks (RRs) or odds ratios (ORs) with corresponding 95\% confidence intervals (CIs) or the numbers of women with PPD were also excluded from this review.

\section{Data extraction and quality assessment}

Data extraction and quality assessment was done by two reviewers independently. Disputes were discussed between the reviewers until consensus was reached. Data extracted from the studies included the first author, year, study sites, sample size, PPD Group, assessment method, quality score, investigation time, as well as risk factors associated with PPD. Articles that fulfilled the predefined criteria were used as a source of data for the final analysis.

The instrument versions were Chinese. The risk factors were assessed by questionnaire and interview. We used the Newcastle-Ottawa Scale (NOS) to assess the quality of the selected cohort and case-control studies [15]. The NOS was used to score the studies on three criteria: the selection of the study groups; the comparability of the groups; and the ascertainment of outcome or exposure. The total score ranged from 0 to 9 , with higher scores representing higher methodological quality and lower risk of bias.

\section{Statistical analysis}

The number of cases of PPD and the number of cases in the control group were converted into the form of odds ratios (ORs) with 95\% confidence intervals (CIs), which were used to pool the outcome data. The Cochrane Q test was performed to assess statistical heterogeneity, and the Higgins $\mathrm{I}^{2}$ statistic was used to determine the extent of variation between effect estimates (0 to 100\%). For outcomes with low heterogeneity, $\mathrm{I}^{2}<50 \%$ and $p>$ 0.1 , the fixed-effects model (M-H method) was used for analysis. In addition to $\mathrm{I}^{2} \geq 50 \%$ or $p<0.1$, the randomeffects model (D-l method) was used [16]. The sensitivity analysis was carried out by changing the model method. Publication bias was evaluated via the visual analysis of funnel plots. In the absence of bias, showing a symmetrical inverted funnel. When drawing a funnel chart, at least 10 studies are needed. The funnel plot may not detect publication bias when the number of studies is small [17]. Statistical analyses were performed using Review Manager 5.3 (Cochrane Collaboration, UK).

\section{Results}

\section{Literature search}

A total of 1175 studies were obtained through six database searches, of which 301 were excluded because of duplicates. Most articles $(n=599)$ were excluded after the title and abstract information were reviewed. Then, 224 articles that did not meet the inclusion criteria were excluded after the full text was reviewed. Finally, the meta-analysis included 51studies, and the study selection process is shown in Fig. 1.

\section{Study characteristics}

A total of 36,705 perinatal women were included across the 12 cohort studies and 39 case-control studies, with 7008 women identified as having PPD. The studies investigated Chinese women in mainland China and Hong Kong, and Canadian immigrants. The EPDS and SelfRating Depression Scale (SDS) were the most common instruments used to assess PPD. The quality score of the included studies ranged from 5 to 8 , and 13 studies were scored 7 or more. The study characteristics and quality evaluation are summarized in Table 1.

\section{Quantitative synthesis \\ Case-control studies and cohort studies}

Seventeen studies (13 case-control studies and 4 retrospective cohort studies) reported that a poor relationship between husband and wife was associated with an increased risk of PPD $\left(\mathrm{OR}=3.56 ; 95 \%\right.$ CI 2.95-4.28; $\mathrm{I}^{2}=$ $30 \% ; p<0.00001$ ) (Fig. 2).

Eleven studies (10 case-control studies, 2 retrospective cohort studies) investigated the association between poor economic foundation and the risk of PPD (OR = 3.67; 95\% CI 3.07-4.37; $\mathrm{I}^{2}=22 \%$; $\mathrm{p}<0.00001$ ) (Fig. 3).

Nine studies (5 case-control studies, 4 retrospective cohort studies) investigated the association between prenatal depression and the risk of PPD (OR $=7.70$; 95\% CI 6.02-9.83; $\mathrm{I}^{2}=69 \% ; p<0.00001$ ) (Fig. 4). Thus, poor economic foundation and prenatal depression were significantly related to PPD.

Nine studies (7 case-control studies, 2 retrospective cohort studies) reported that a poor relationship between mother-in-law and daughter-in-law was associated with an increased risk of PPD (OR $=2.89$; 95\% CI 2.123.95; $\mathrm{I}^{2}=72 \% ; p<0.00001$ ) (Fig. 5).

Nine studies (4 case-control studies, 5 retrospective cohort studies) investigated the association between a lack of social support and the risk of PPD $(\mathrm{OR}=2.66$; 95\% CI 1.57-4.53; $\left.\mathrm{I}^{2}=98 \% ; p=0.0003\right)$. After subgroup analysis according to the type of study design, the heterogeneity of the case-control group decreased $\left(\mathrm{I}^{2}=46 \%\right.$, $p=0.14$ ), but the heterogeneity of the cohort group was still very high $\left(\mathrm{I}^{2}=99 \%, p<0.00001\right)$. The heterogeneity of the cohort group decreased significantly when one 


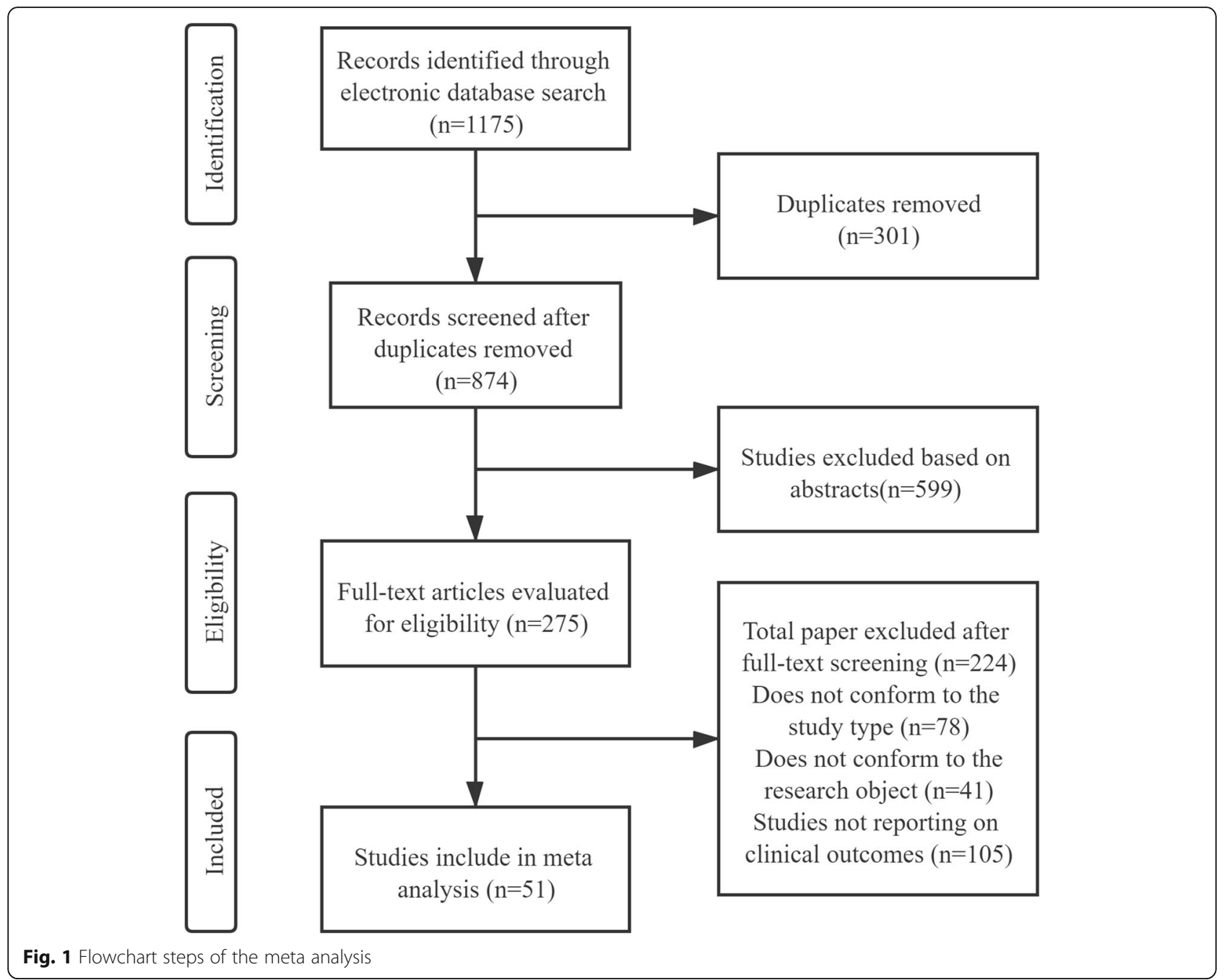

study was excluded [67]. ( $\left.\mathrm{I}^{2}=42 \%, p=0.16\right)$ (Fig. 6, 7). This may be due to major differences in social support between Chinese-Canadian women and Chinese women [26].

\section{Case-control studies}

Seven studies investigated the association between unplanned pregnancy and the risk of PPD. The pooled OR for unplanned pregnancy was 2.55 (95\% CI 2.08-3.14; $\mathrm{I}^{2}=26 \% ; p<0.00001$ ) (Fig. 8). Six studies investigated the association between poor living conditions and the risk of PPD. The pooled OR for poor living conditions was 2.44 (95\% CI $\left.1.92-3.10 ; \quad \mathrm{I}^{2}=21 \% ; \quad p<0.00001\right)$ (Fig. 9). Four studies investigated the association between prenatal anxiety and the risk of PPD. The pooled OR for prenatal anxiety was 7.07 (95\% CI 4.12-12.13; $\left.\mathrm{I}^{2}=59 \% ; p<0.00001\right)$, and a random-effects model was adopted (Fig. 10). Five studies investigated the association between mothers-in-law as caregivers and the risk of PPD. The pooled OR for mothers-in-law as caregivers was $1.95\left(95 \%\right.$ CI $\left.1.54-2.48 ; \mathrm{I}^{2}=33 \% ; \quad p<0.00001\right)$ (Fig. 11).

Therefore, unplanned pregnancy, poor living conditions, prenatal anxiety, and mothers-in-law as caregivers were found to be significantly associated with PPD.

\section{Heterogeneity test and sensitivity analysis}

The results of 51 studies were tested by the Cochrane Q test. The heterogeneity test results of the seven risk factors were low or medium (prenatal depression, poor economic foundation, poor relationship with partner, lack of social support, unplanned pregnancy, mothers-in-law as caregivers, and poor living conditions). However, for the poor mother-in-law relationship and prenatal anxiety, the random-effects model was used because of the high heterogeneity. Among them, the high heterogeneity of the poor relationship between mother-in-law and daughter-in-law may be due to the lack of targeted 
Table 1 Characteristics of included studies

\begin{tabular}{|c|c|c|c|c|c|c|c|c|}
\hline $\begin{array}{l}\text { First } \\
\text { author }\end{array}$ & Year & study sites & $\begin{array}{l}\text { Sample } \\
\text { size }\end{array}$ & $\begin{array}{l}\text { PPD } \\
\text { Group }\end{array}$ & $\begin{array}{l}\text { Assessment } \\
\text { method }\end{array}$ & $\begin{array}{l}\text { Quality } \\
\text { scores }\end{array}$ & Investigate time & $\begin{array}{l}\text { Risk } \\
\text { factors }\end{array}$ \\
\hline Lee [18] & 2004 & Hong Kong & 781 & 122 & EPDS & 8 & first trimester/ 6 weeks postpartum & $(1),(5)$ \\
\hline Zhao [19] & 2018 & Shanghai & 215 & 67 & EPDS & 7 & $\begin{array}{l}\text { late pregnancy/ week 3-days and 6-weeks after } \\
\text { delivery }\end{array}$ & (1) \\
\hline Pan [20] & 2004 & Sichuan & 427 & 33 & EPDS & 6 & third trimester/ $4 \sim 6$ weeks postpartum & $(1),(3)$ \\
\hline Zhao [21] & 2018 & Sichuan & 1440 & 25 & EPDS & 6 & third trimester/ 8,18 weeks postpartum & (1) \\
\hline Siu [18] & 2012 & Hong Kong & 805 & 126 & EPDS & 7 & third trimester/ around 2 months postnatally & $(4),(5)$ \\
\hline Li [22] & 2017 & Shanxi & 1759 & 593 & EPDS & 6 & third trimester/ 4 weeks postpartum & $(4),(6)$ \\
\hline Gu [23] & 2004 & Shanghai & 999 & 307 & HADS & 6 & Second trimester/ 4 weeks postpartum & (3),(4) \\
\hline Sun [24] & 2015 & Yunnan & 528 & 96 & EPDS & 6 & third trimester/ 4 weeks postpartum & (6) \\
\hline Kang [25] & 2015 & Jiangsu & 3972 & 468 & EPDS $>10$ & 6 & first trimester/ 6 weeks postpartum & (6) \\
\hline Dennis [26] & 2017 & $\begin{array}{l}\text { Canadian } \\
\text { immigrants }\end{array}$ & 549 & 120 & $E P D S>9$ & 8 & third trimestery/ 4 weeks postpartum & (6) \\
\hline Cai [27] & 2017 & Chongqing & 371 & 60 & EPDS $\geq 13$ & 7 & Second trimester/ 6 weeks postpartum & (6) \\
\hline $\mathrm{Hu}[28]$ & 2010 & Sichuan & 264 & 146 & $E P D S>9$ & 6 & Second trimester/ 4 weeks postpartum & (4) \\
\hline Gu [29] & 2017 & Xinjiang & 824 & 286 & EPDS $>13$ & 6 & 42 days postpartum & $(1),(2)$ \\
\hline Zhang [30] & 2017 & Guangdong & 538 & 49 & $E P D S>10$ & 7 & 42 days postpartum & $(1),(2)$ \\
\hline Wu [31] & 2019 & Guangdong & 1437 & 100 & EPDS $>10$ & 6 & 4 weeks postpartum & $(1),(2)$ \\
\hline Shen [32] & 2011 & Shanxi & 104 & 52 & EPDS $>13$ & 6 & 4 weeks postpartum & (1) \\
\hline Yang [33] & 2016 & Hubei & 400 & 37 & EPDS $>13$ & 6 & $4 \sim 6$ weeks postpartum & $(1),(3),(8)$ \\
\hline Zhang [34] & 2001 & Tianjin & 463 & 47 & $E P D S \geq 13$ & 7 & 4 weeks postpartum & (4) \\
\hline Yin [35] & 2011 & Guangdong & 202 & 37 & $\mathrm{SDS}>40$ & 5 & 4 weeks postpartum & (3),(4) \\
\hline Huang [36] & 2012 & Hunan & 302 & 56 & EPDS & 6 & $6 \sim 7$ weeks postpartum & (4) \\
\hline Song [37] & 2012 & Hunan & 285 & 69 & SDS & 5 & 4 weeks postpartum & (3), (4), (5) \\
\hline Wang [38] & 2013 & Beijing & 435 & 27 & $\mathrm{SCL}-90>2$ & 6 & 42 days postpartum & (4) \\
\hline Lin [39] & 2014 & Zhejiang & 2023 & 204 & EPDS & 5 & 42 days postpartum & (4) \\
\hline Zhou [40] & 2014 & Hubei & 378 & 294 & EPDS/SDS & 5 & $4 \sim 6$ weeks postpartum & (4) \\
\hline Wang [41] & 2014 & Guizhou & 875 & 112 & EPDS & 7 & 42 days postpartum & (3),(4),(8) \\
\hline Chen [42] & 2017 & Zhejiang & 380 & 260 & EPDS $>13$ & 7 & 42 days postpartum & $(4),(8)$ \\
\hline Jiang [43] & 2018 & Shandong & 185 & 25 & EPDS & 5 & 6 weeks postpartum & (4) \\
\hline Han [44] & 2018 & Henan & 248 & 124 & EPDS $>10$ & 5 & 4 weeks postpartum & (4) \\
\hline $\mathrm{He}$ [45] & 2019 & Zhejiang & 398 & 217 & SDS EPDS & 5 & 4 weeks postpartum & (4) \\
\hline Yu [46] & 2010 & Shanghai & 673 & 73 & EPDS & 7 & 6 weeks postpartum & $(3),(7)$ \\
\hline Zhang [47] & 2012 & Hunan & 215 & 67 & EPDS $>13$ & 6 & $30 \sim 42$ days postpartum & (7) \\
\hline Zhang [48] & 2014 & Guangdong & 586 & 87 & EPDS $>13$ & 6 & 6 weeks postpartum & (7) \\
\hline Li [49] & 2014 & Anhui & 687 & 103 & EPDS & 7 & 6 weeks postpartum & $(3),(7)$ \\
\hline Liu [50] & 2015 & Hunan & 232 & 43 & EPDS/HAD & 5 & 42 days postpartum & (7) \\
\hline Liu [51] & 2015 & Heilongjiang & 576 & 162 & EPDS & 6 & 42 days postpartum & $(6),(7)$ \\
\hline Chen [52] & 2018 & Northwest China & 640 & 84 & EPDS $>13$ & 6 & 6 weeks postpartum & $(7),(9)$ \\
\hline Wang [53] & 2013 & Shandong & 917 & 168 & EPDS/SDS & 5 & 6 weeks postpartum & (3) \\
\hline Li [54] & 2019 & Shanxi & 170 & 85 & SDS & 5 & 6 weeks postpartum & $(3),(6)$ \\
\hline Guan [55] & 2012 & Inner Mongolia & 246 & 92 & $E P D S>9$ & 6 & $30 \sim 42$ days postpartum & (9) \\
\hline Zhang [56] & 2011 & Hubei & 479 & 167 & $\mathrm{BDI}>=5$ & 6 & $7 \sim 30$ days postpartum & (9) \\
\hline Deng [57] & 2014 & Guangdong & 2021 & 158 & CES-D $>20$ & 6 & 42 days postpartum & (5) \\
\hline Deng [4] & 2014 & Guangdong & 1823 & 499 & EPDS $>13$ & 7 & 4 weeks postpartum & (5) \\
\hline Han [58] & 2015 & Beijing & 203 & 189 & $\begin{array}{l}E P D S>13 \\
H A D>9\end{array}$ & 6 & 42 days postpartum & $(5),(8)$ \\
\hline Liu [59] & 2017 & Guangdong & 418 & 93 & EPDS $>9.5$ & 5 & $4 \sim 6$ weeks postpartum & (5) \\
\hline Zhou [60] & 2019 & Jiangsu & 849 & 142 & EPDS $>10$ & 6 & $2 \sim 6$ weeks postpartum & (5) \\
\hline
\end{tabular}


Table 1 Characteristics of included studies (Continued)

\begin{tabular}{|c|c|c|c|c|c|c|c|c|}
\hline $\begin{array}{l}\text { First } \\
\text { author }\end{array}$ & Year & study sites & $\begin{array}{l}\text { Sample } \\
\text { size }\end{array}$ & $\begin{array}{l}\text { PPD } \\
\text { Group }\end{array}$ & $\begin{array}{l}\text { Assessment } \\
\text { method }\end{array}$ & $\begin{array}{l}\text { Quality } \\
\text { scores }\end{array}$ & Investigate time & $\begin{array}{l}\text { Risk } \\
\text { factors }\end{array}$ \\
\hline Liu [61] & 2015 & Hubei & 1427 & 198 & $E P D S \geq 9$ & 5 & 42 days postpartum & $(6),(8)$ \\
\hline Xie [62] & 2018 & Hubei & 534 & 103 & EPDS & 6 & 6 weeks postpartum & (6) \\
\hline Pan [63] & 2015 & Zhejiang & 745 & 93 & EPDS & 5 & 42 days postpartum & $(2)$ \\
\hline Yang [64] & 2020 & Jiangxi & 371 & 94 & EPDS & 6 & 42 days postpartum, third trimester, & $(3),(4),(9)$ \\
\hline Huang [65] & 2020 & Jiangsu & 784 & 59 & EPDS & 6 & 42 days postpartum & (3) \\
\hline Qing [66] & 2020 & Guangdong & 522 & 90 & EPDS & 7 & 2nd to 4 th postnatal months & (9) \\
\hline
\end{tabular}

Risk factors: (1) Prenatal depression, (2) prenatal anxiety, (3) poor economic foundation, (4) poor relationship between husband and wife, (5) poor relationship between mother-in-law and daughter-in-law, (6) lack of social support, (7) unplanned pregnancy and (8) poor living conditions, (9) mother-in-law as the caregiver PPD postpartum depression, EPDS Edinburgh postnatal depression scale, HADS Hospital Anxiety and Depression Scale, SDS self-rating depression scale, SCL-90 symptom checklist 90, BDI beck depression inventory, CES-D Center for Epidemiological Studies Depression Scale

questionnaire surveys, the setting of the questionnaires being more subjective, and the measurement standard more difficult to unify. The high heterogeneity of prenatal anxiety may be due to the different times of prenatal measurement [17]. In sensitivity analyses, the change model method was used to estimate the point and interval of the OR values of all risk factors to judge the stability of the meta-analysis (Table 2). The point estimates of the combined OR values of the fixed-effects model and the random-effects model were similar, and the interval estimation range of the random-effects model was slightly wider than that of the fixed-effects model. This result indicated that the comprehensive analysis results of the influencing factors in this study were reliable overall.

\section{Publication bias}

Publication bias was evaluated via the visual analysis of funnel plots. The funnel plot generally appeared to be symmetrical, indicating no publication bias (Fig. 12, 13).

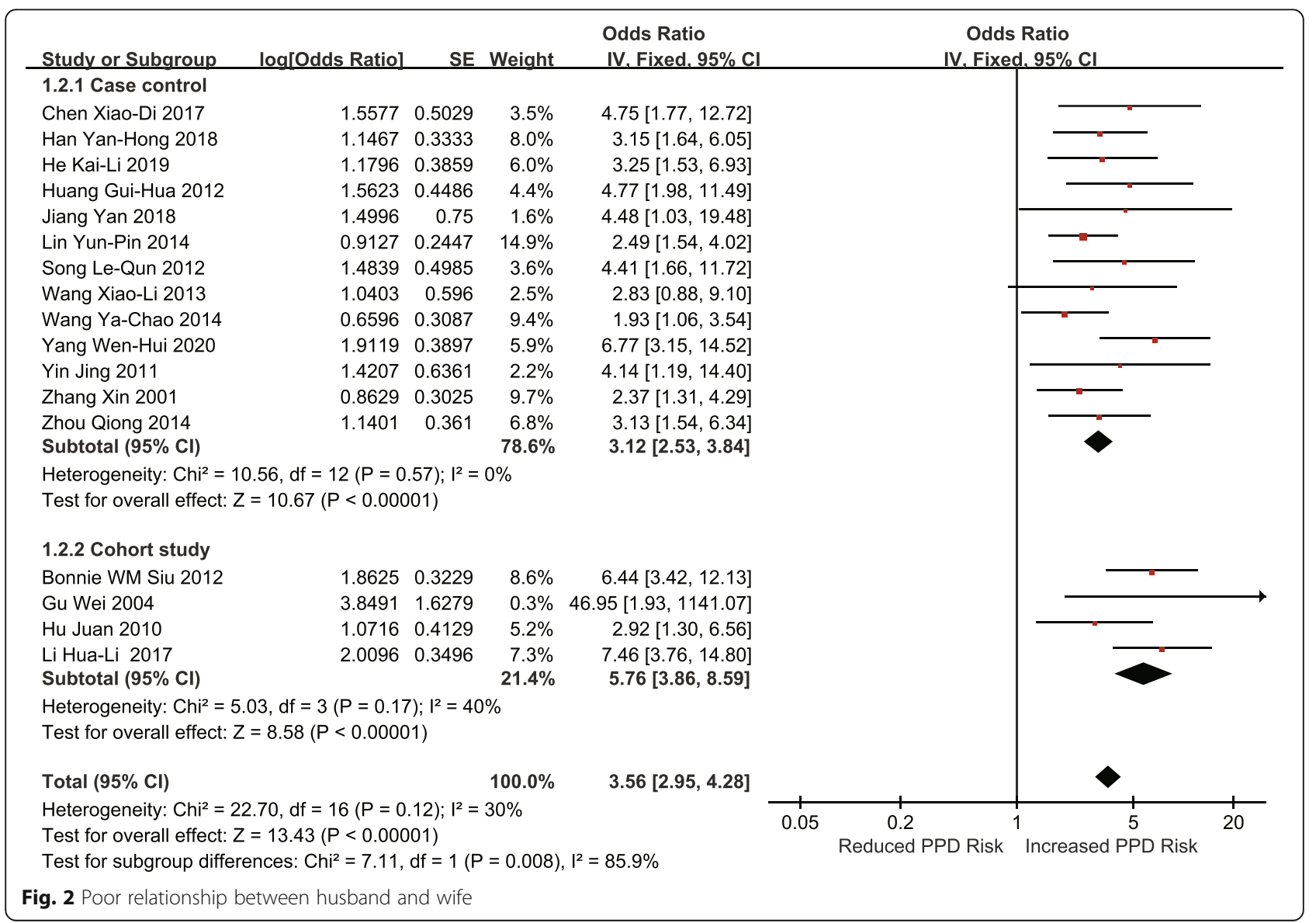




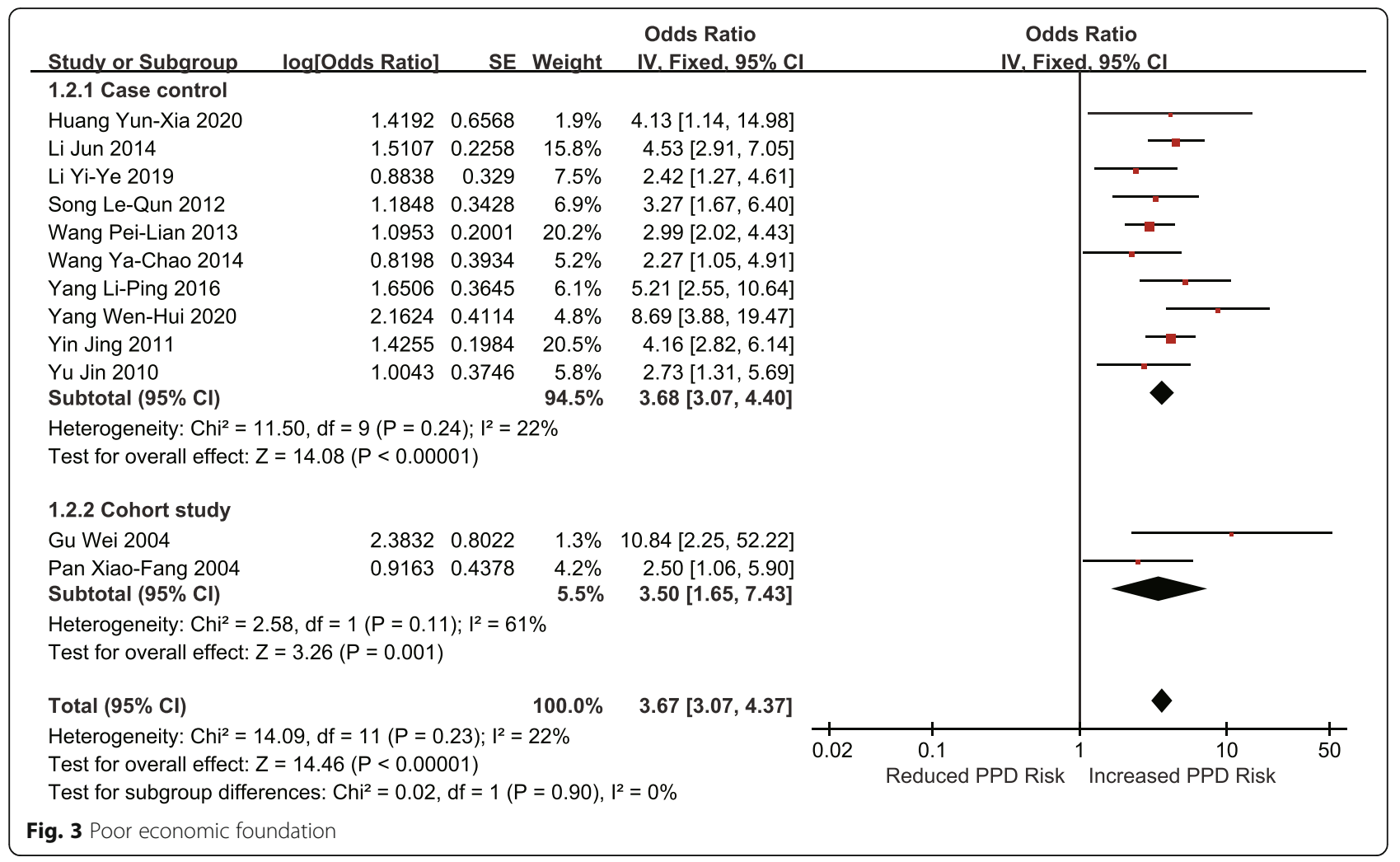

\section{Discussion}

PPD is a crucial part of the spectrum of mood disturbances affecting postpartum women. A variety of factors affect the physical and mental health of pregnant women. Thus, identifying alterable risk factors for PPD and controlling them at an early stage are essential for the treatment and prevention of this condition.

The psychosocial risk factors for PPD in Chinese women identified in this meta-analysis mainly included three kinds: prenatal emotional factors (prenatal anxiety

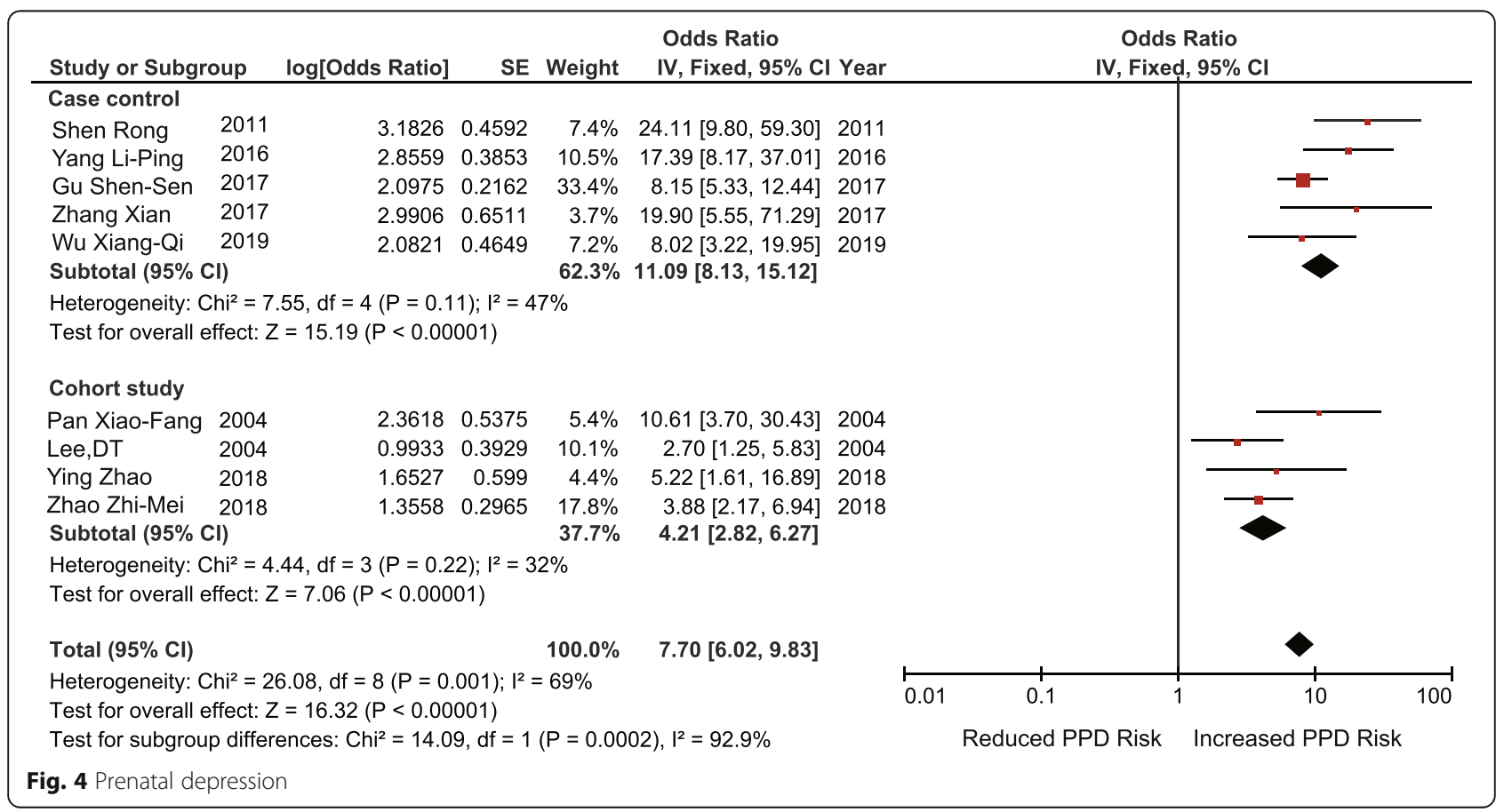




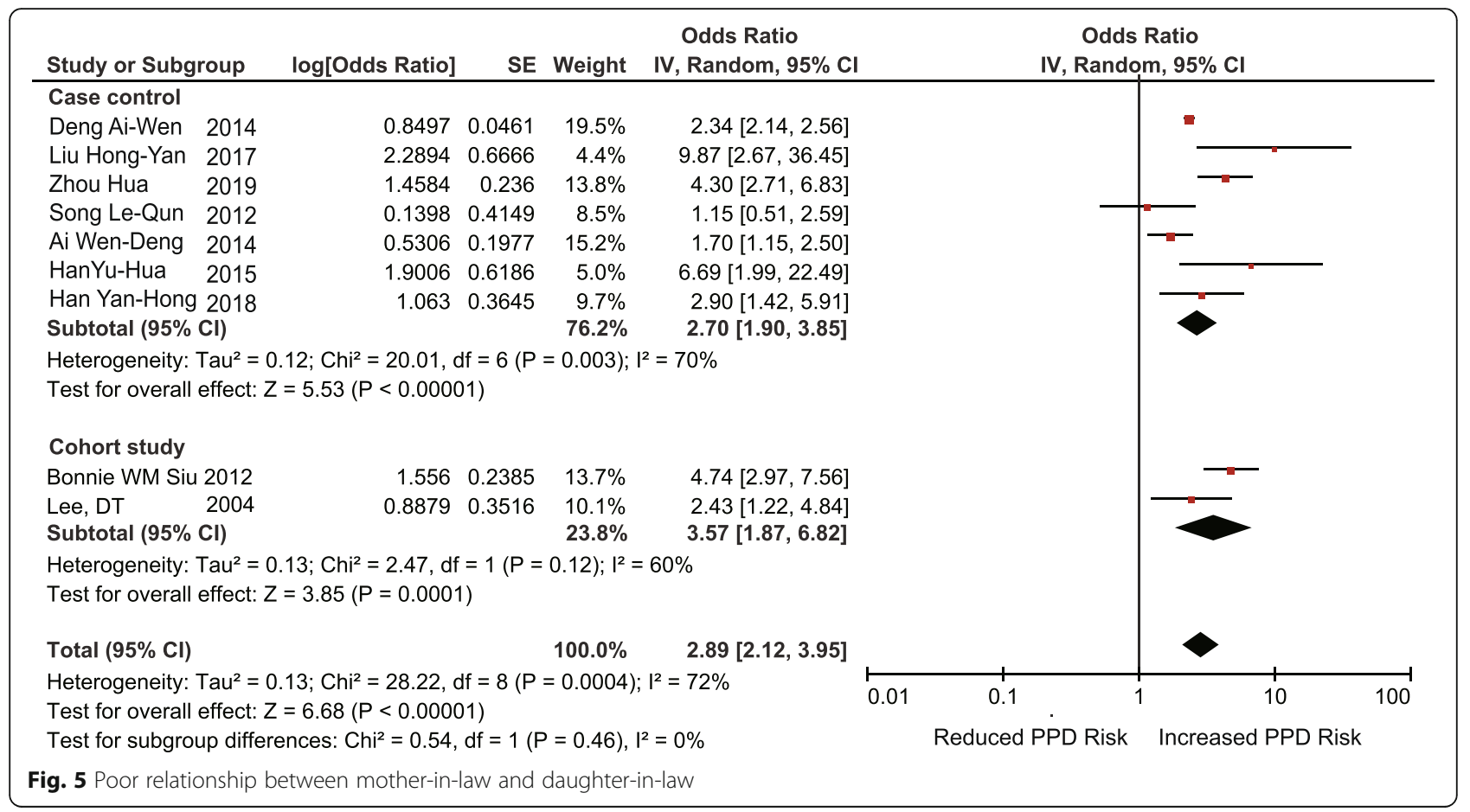

and prenatal depression), social demographic factors (poor marital relationship, poor living conditions, lack of social support, and unplanned pregnancy) and social and interpersonal factors (poor relationship between husband and wife, poor relationship between mother-in-law and daughter-in-law,and mother-in-law as the caregiver).
First, prenatal anxiety and depression were significantly associated with an increased risk of PPD, as confirmed by some Western studies. An Italian study [68] showed that women with depression or anxiety during pregnancy and a lack of support from family and friends were at a higher risk of PPD. This result has also been

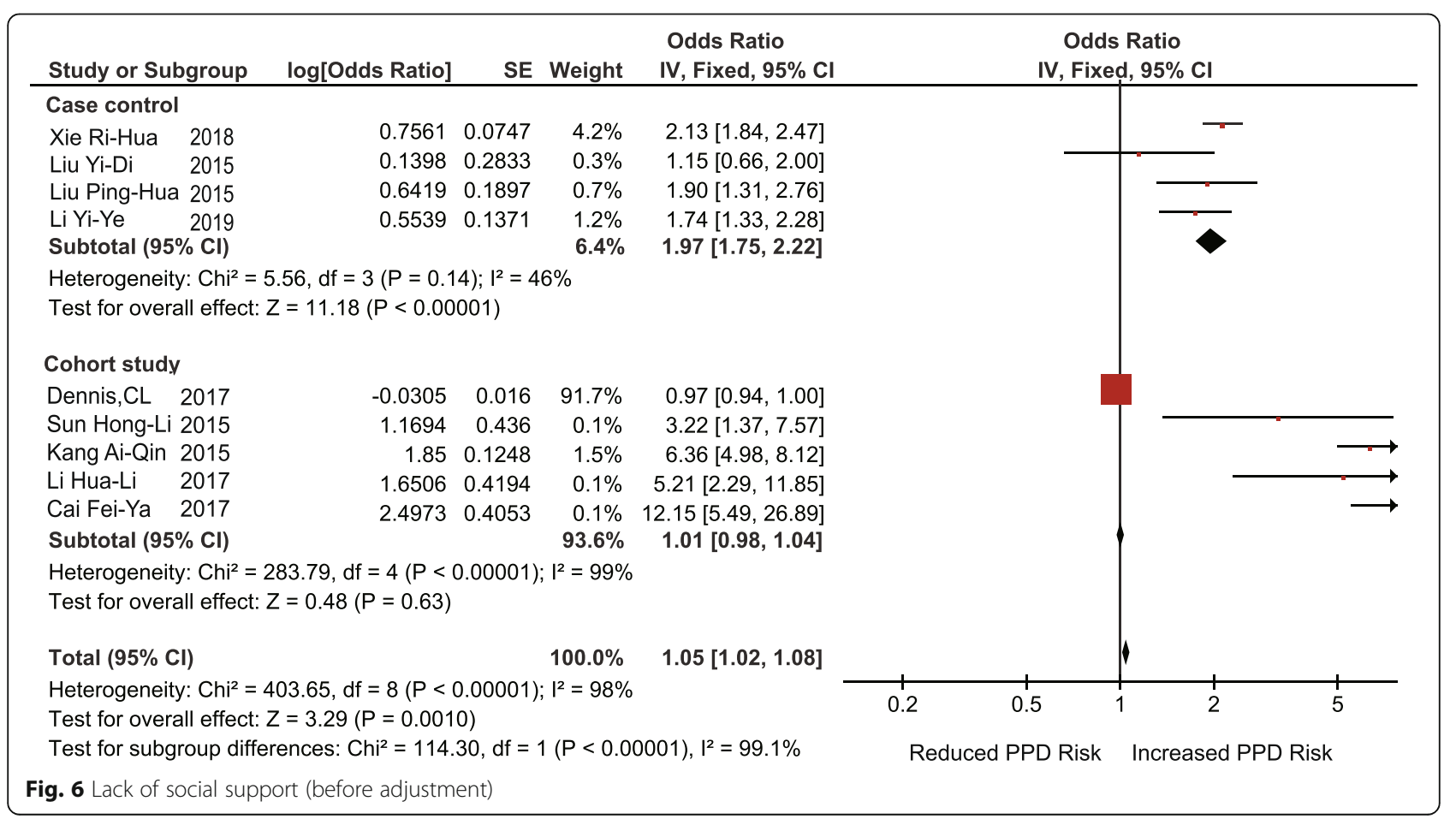




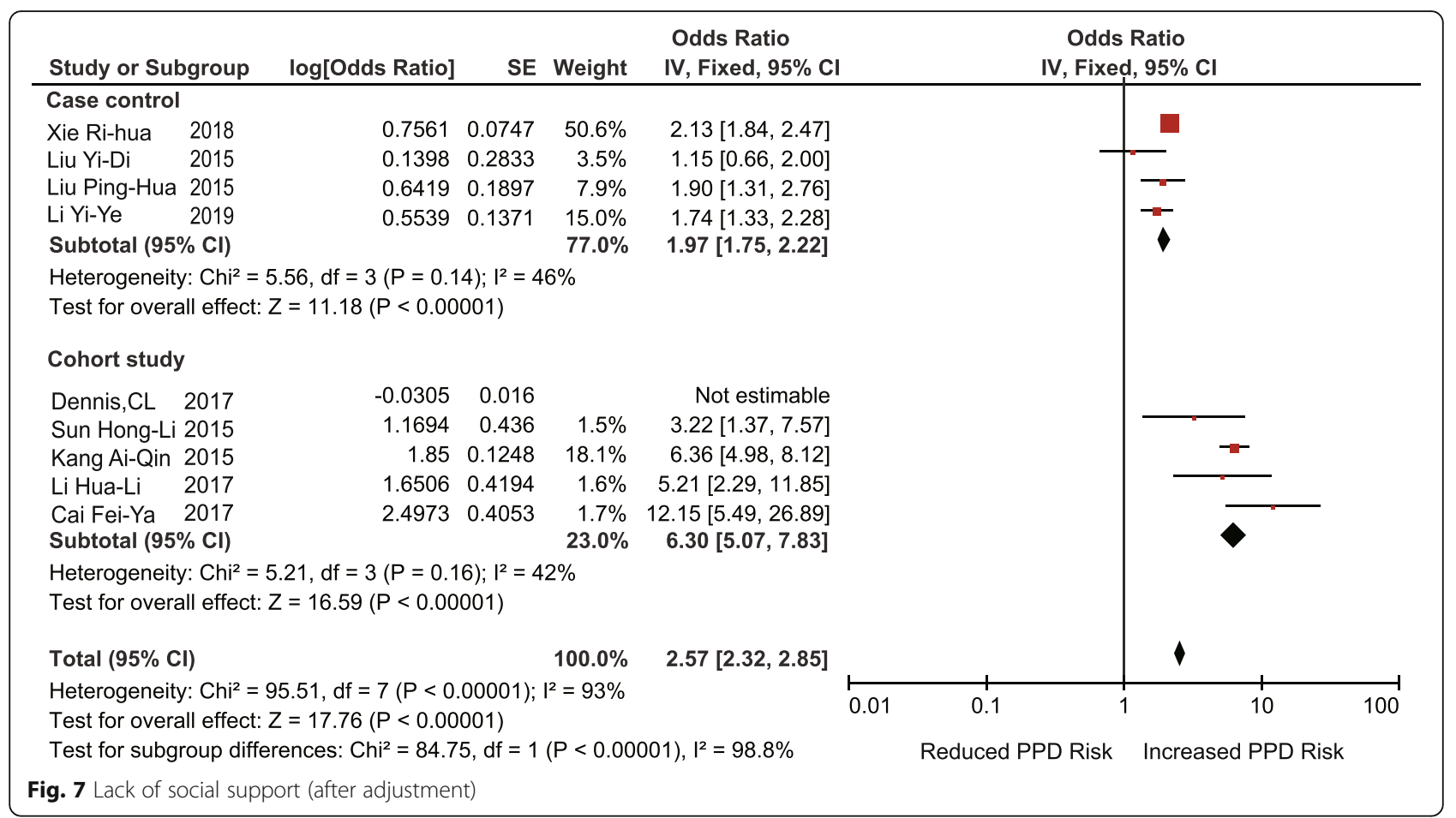

confirmed in China. According to the report of Lee [69], most PPD was the continuation of prenatal psychological problems and emotional disorders, indicating a significant correlation between prenatal psychological status and the occurrence of PPD.

Another explanation for the effect of prenatal emotional distress is physiological changes. For example, excessive anxiety and depression in pregnant women may lead to a series of physiological and pathological reactions, such as a decrease in norepinephrine secretion and changes in other endocrine hormones, which may lead to the weakening of uterine contractions, a prolonged stage of labor, and increased bleeding. These challenges further aggravate the anxiety of pregnant women and lead to an increased risk of developing PPD [70].

Second, this study found that social demographic factors were also risk factors for PPD, such as a poor economic foundation, poor living conditions, a lack of social support, and unplanned pregnancy. Among them, the economic foundation of the family had an important effect on the psychological status of the mother. Previous reviews suggested that the status of the family's economic income was positively related to the level of stress in pregnant women. $\mathrm{Yu}$ [46] suggested that after adjustments were made for other related factors, the incidence of PPD among women who were worried about family economic status was 3.162 times higher than among

\begin{tabular}{|c|c|c|c|c|c|c|c|}
\hline \multicolumn{2}{|c|}{ Study or Subgroup } & log[Odds Ratio] & \multicolumn{2}{|c|}{ SE Weight } & $\begin{array}{l}\text { Odds Ratio } \\
\text { IV, Fixed, } 95 \% \text { CI Year }\end{array}$ & \multicolumn{2}{|c|}{$\begin{array}{c}\text { Odds Ratio } \\
\text { IV. Fixed, } 95 \% \mathrm{Cl}\end{array}$} \\
\hline Yu Jin & 2010 & 1.1227 & 0.4663 & $5.2 \%$ & $3.07[1.23,7.66] 2010$ & & \\
\hline Yong Zhang & 2012 & 1.4693 & 0.6103 & $3.0 \%$ & $4.35[1.31,14.37] 2012$ & & \\
\hline Zhang & 2014 & 1.1119 & 0.2452 & $18.6 \%$ & $3.04[1.88,4.92] 2014$ & & $\longrightarrow$ \\
\hline Li Jun & 2014 & 1.1537 & 0.2199 & $23.2 \%$ & $3.17[2.06,4.88] 2014$ & & $\rightarrow$ \\
\hline Liu Shen-Mei & 2015 & 1.5282 & 0.4971 & $4.5 \%$ & $4.61[1.74,12.21] 2015$ & & \\
\hline Liu Yi-Di & 2015 & 0.5365 & 0.198 & $28.6 \%$ & $1.71[1.16,2.52] 2015$ & & $\rightarrow-$ \\
\hline Ling Chenn & 2018 & 0.8185 & 0.2577 & $16.9 \%$ & $2.27[1.37,3.76] 2018$ & & - \\
\hline \multicolumn{3}{|c|}{ Total $(95 \% \mathrm{Cl})$} & & $100.0 \%$ & $2.55[2.08,3.14]$ & & $\nabla$ \\
\hline \multicolumn{5}{|c|}{$\begin{array}{l}\text { Heterogeneity: } \mathrm{Ch}^{2}=8.12, \mathrm{df}=6(P=0.23) ;\left.\right|^{2}=26 \% \\
\text { Test for overall effect: } Z=8.86(P<0.00001)\end{array}$} & & $\begin{array}{ll}0.01 & 0.1 \\
\text { Reduced PPD Risk } & 1\end{array}$ & $\begin{array}{c}10 \\
\text { Increased PPD Risk }\end{array}$ \\
\hline Fig. 8 Unplanned & regnanc & & & & & & \\
\hline
\end{tabular}




\begin{tabular}{|c|c|c|c|c|c|c|c|}
\hline \multicolumn{2}{|c|}{ Study or Subgroup } & log[Odds Ratio] & \multicolumn{2}{|c|}{ SE Weight } & Odds Ratio & \multicolumn{2}{|c|}{$\begin{array}{c}\text { Odds Ratio } \\
\text { IV. Fixed, } 95 \% \mathrm{Cl} \\
\end{array}$} \\
\hline WangYa-Chao & 2014 & 0.9286 & 0.385 & $9.9 \%$ & $2.53[1.19,5.38] 2014$ & & \\
\hline Liu Ping-Hua & 2015 & 0.6481 & 0.1634 & $55.2 \%$ & $1.91[1.39,2.63] 2015$ & & - \\
\hline Han Yu-Hua & 2015 & 1.5063 & 0.5482 & $4.9 \%$ & $4.51[1.54,13.21] 2015$ & & \\
\hline Pan Li-Sha & 2015 & 1.3499 & 0.5467 & $4.9 \%$ & $3.86[1.32,11.26] 2015$ & & \\
\hline Yang Li-Ping & 2016 & 1.3838 & 0.3524 & $11.9 \%$ & $3.99[2.00,7.96] 2016$ & & \\
\hline Chen Xiao-Di & 2017 & 1.0463 & 0.3341 & $13.2 \%$ & $2.85[1.48,5.48] 2017$ & & \\
\hline & & $100.0 \%$ & $2.44[1.92,3.10]$ & & 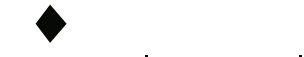 \\
\hline \multicolumn{5}{|c|}{$\begin{array}{l}\text { Heterogeneity: } C i^{2}=6.36, d f=5(P=0.27) ; I^{2}=21 \% \\
\text { Test for overall effect: } Z=7.35(P<0.00001)\end{array}$} & & $0.01 \quad 0.1$ & $\begin{array}{cc}10 & 100 \\
\text { Increased PPD Risk }\end{array}$ \\
\hline Fig. 9 Poor living $c$ & indition & & & & & & \\
\hline
\end{tabular}

those who did not worry about it. The probable explanation may be that after childbirth, the cost of raising the baby and the basic cost of living for the family significantly increases. If the family income is insufficient, it will lead to high levels of pressure for pregnant women and can easily cause negative emotions. In recent years, with China's two-child policy, raising multiple children in a family increases the family's financial burden, which may be a factor of PPD. A study in Turkey shows that there was a significant relationship between monthly income and depression, which was similar to the results of the present study [71].

In addition, previous studies have shown that social support was a protective factor against PPD, and as far as mothers were concerned, the greatest social support comes from their husbands. Xiong et al. [72] suggested that puerperae with spousal support were much less likely to develop PPD. A Chinese study also confirmed that high levels of social support can reduce the risk of PPD, with other factors were fixed [27]. Our findings are generally consistent with those of previous reviews. Therefore, giving adequate social support to parturients during the puerperal period can help them get through this critical period smoothly.
Third, in this study, the interpersonal risk factors for PPD were a poor relationship between husband and wife, a poor relationship between mother-in-law and daughter-in-law, and mother-in-law as the caregiver. The poor relationship between husbands and wives, as an important factor affecting human physical and mental health, has attracted the close attention of researchers worldwide. Zhang [73] suggested that the quality of the husband-wife relationship was mainly reflected in the quality of the husband's care for his wife, and women who were less satisfied with their husband's care were more likely to have depression. Poor marriage and family relationships will not only reduce maternal social support but also become a maternal stressful life event, which brings about an increased risk of developing PPD. This study was confirmed in a Polish study. Malus et al. [74] confirmed the significance of the marital relationship in the development of PPD. A sense of closeness and intimacy in the relationship were associated with better mood and a greater ability to cope with the difficulties of labor, puerperium, and caring for a newborn baby.

In addition, the results of this study found that the risk factors for PPD related to Chinese cultural

\begin{tabular}{|c|c|c|c|c|c|c|c|}
\hline Study or Subgroup & \multicolumn{2}{|c|}{ log[Odds Ratio] } & \multicolumn{2}{|c|}{ SE Weight } & $\begin{array}{l}\text { Odds Ratio } \\
\text { IV. Random. } 95 \% \text { CI Year }\end{array}$ & \multicolumn{2}{|c|}{$\begin{array}{c}\text { Odds Ratio } \\
\text { IV, Random, } 95 \% \mathrm{Cl}\end{array}$} \\
\hline Gu Shen-Sen & 2017 & 2.2908 & 0.2417 & $32.8 \%$ & $9.88[6.15,15.87] 2017$ & & $\rightarrow-$ \\
\hline Zhang Xian & 2017 & 1.4796 & 0.5799 & $14.9 \%$ & $4.39[1.41,13.68] 2017$ & & \\
\hline Chen Li-Shan & 2018 & 2.3933 & 0.3044 & $28.5 \%$ & $10.95[6.03,19.88] 2018$ & & \\
\hline Wu Xiang-Qi & 2019 & 1.27 & 0.3812 & $23.8 \%$ & $3.56[1.69,7.52] 2019$ & & \\
\hline Total $(95 \% \mathrm{Cl})$ & & & & $100.0 \%$ & $7.07[4.12,12.13]$ & & \\
\hline \multicolumn{6}{|c|}{$\begin{array}{l}\text { Heterogeneity: } \text { Tau }^{2}=0.17 ; \mathrm{Chi}^{2}=7.40, \mathrm{df}=3(P=0.06) ; I^{2}=59 \% \\
\text { Test for overall effect: } Z=7.10(P<0.00001)\end{array}$} & $\begin{array}{l}0.01 \\
\text { Reduced PPD Risk }\end{array}$ & $\begin{array}{cc}10 & 100 \\
\text { Increased PPD Risk }\end{array}$ \\
\hline \multicolumn{6}{|c|}{ Fig. 10 Prenatal anxiety } & & \\
\hline
\end{tabular}




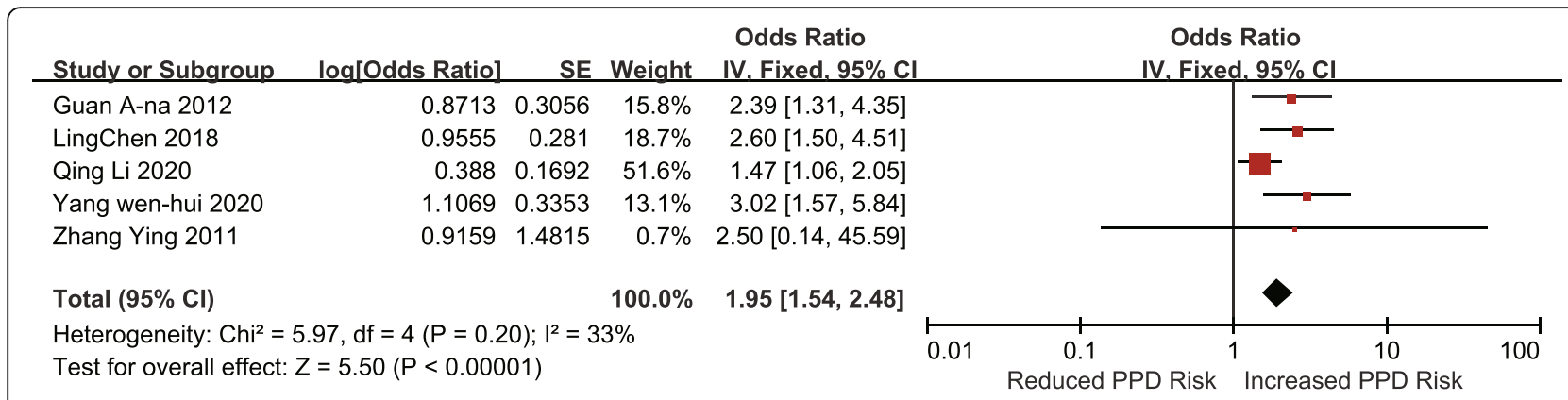

Fig. 11 Mother-in-law as the caregiver

characteristics included the mother-in-law as the caregiver and a poor relationship between mother-in-law and daughter-in-law.

From the perspective of family structure, Chinese families have a close relationship, intergenerational support shows a circular mode, and it is very common to expand families, especially families with three generations living together. Even if the whole family emigrates to other countries, it is still common for parents to live together with their children's families. The kinship network linked by blood relationship is complicated, and the conflict increases accordingly [75].

In Western countries, nuclear families are the main organizational form; they attach importance to the development of personality, pay attention to independence and privacy, and think that the lifestyle of several generations living together seriously infringes upon the privacy of individuals, which is unbearable. Therefore, adult children live independently of their parents. Kinship is more distant than that of China, and contradictions and intergenerational conflicts are correspondingly less [76].

Traditionally, mothers-in-law exercise significant power in the family and are a major influence on the postpartum care of new mothers. In China, due to the influence of doing-the-month culture, mothers and newborns are mostly cared for by their mothersin-law. The strain between mothers-in-law and daughters-in-law is a sensitive problem and may be a cause of PPD in China. Steinberg [77] indicated that the strain between mothers-in-law and daughters-inlaw often offset the benefits of assistance and may even contribute to negative mood during the postpartum period. In traditional Confucian philosophy, the new mother should be considered a good daughterin-law if she behaves in a way that is respectful at home and is obedient to her in-laws and husband [78]. The relationship between women and their

Table 2 Sensitivity analysis of risk factors of PPD

\begin{tabular}{|c|c|c|c|c|c|}
\hline \multirow[b]{2}{*}{ Risk factors } & \multirow[b]{2}{*}{ Type of research } & \multicolumn{2}{|c|}{ Fixed effect model } & \multicolumn{2}{|c|}{ Random effect model } \\
\hline & & $\mathrm{OR}$ & $95 \% \mathrm{Cl}$ & OR & $95 \% \mathrm{Cl}$ \\
\hline \multirow[t]{2}{*}{ Prenatal depression } & Cohort study & 4.21 & {$[2.82,6.27]$} & 4.40 & {$[2.64,7.34]$} \\
\hline & Case control study & 11.09 & {$[8.13,15.12]$} & 12.72 & {$[7.85,20.62]$} \\
\hline \multirow[t]{2}{*}{ marriage relationship } & Cohort study & 5.76 & {$[3.86,8.59]$} & 5.76 & {$[3.27,10.13]$} \\
\hline & Case control study & 3.12 & {$[2.53,3.84]$} & 3.12 & {$[2.53,3.84]$} \\
\hline \multirow[t]{2}{*}{ Mother-in-law relationship } & Cohort study & 3.84 & {$[2.61,5.65]$} & 3.57 & {$[1.87,6.82]$} \\
\hline & Case control study & 2.37 & {$[2.17,2.58]$} & 2.70 & {$[1.90,3.85]$} \\
\hline \multirow[t]{2}{*}{ Social support } & Cohort study & 6.30 & {$[5.07,7.83]$} & 6.18 & {$[4.10,9.31]$} \\
\hline & Case control study & 1.97 & {$[1.75,2.22]$} & 1.85 & {$[1.52,2.25]$} \\
\hline \multirow[t]{2}{*}{ Economic foundation } & Cohort study & 3.50 & {$[1.65,7.43]$} & 3.67 & {$[2.97,4.55]$} \\
\hline & Case control study & 3.68 & {$[3.07,4.40]$} & 4.46 & {$[1.09,8.20]$} \\
\hline Unplanned pregnancy & Case control study & 2.55 & {$[2.08,3.14]$} & 2.64 & {$[2.04,3.40]$} \\
\hline Prenatal anxiety & Case control study & 7.93 & {$[5.76,10.90]$} & 7.07 & {$[4.12,12.13]$} \\
\hline Mother-in-law as the caregiver & Case control study & 1.95 & {$[1.54,2.48]$} & 2.11 & {$[1.52,2.93]$} \\
\hline Living conditions & Case control study & 2.44 & {$[1.92,3.10]$} & 2.65 & {$[1.96,3.57]$} \\
\hline
\end{tabular}




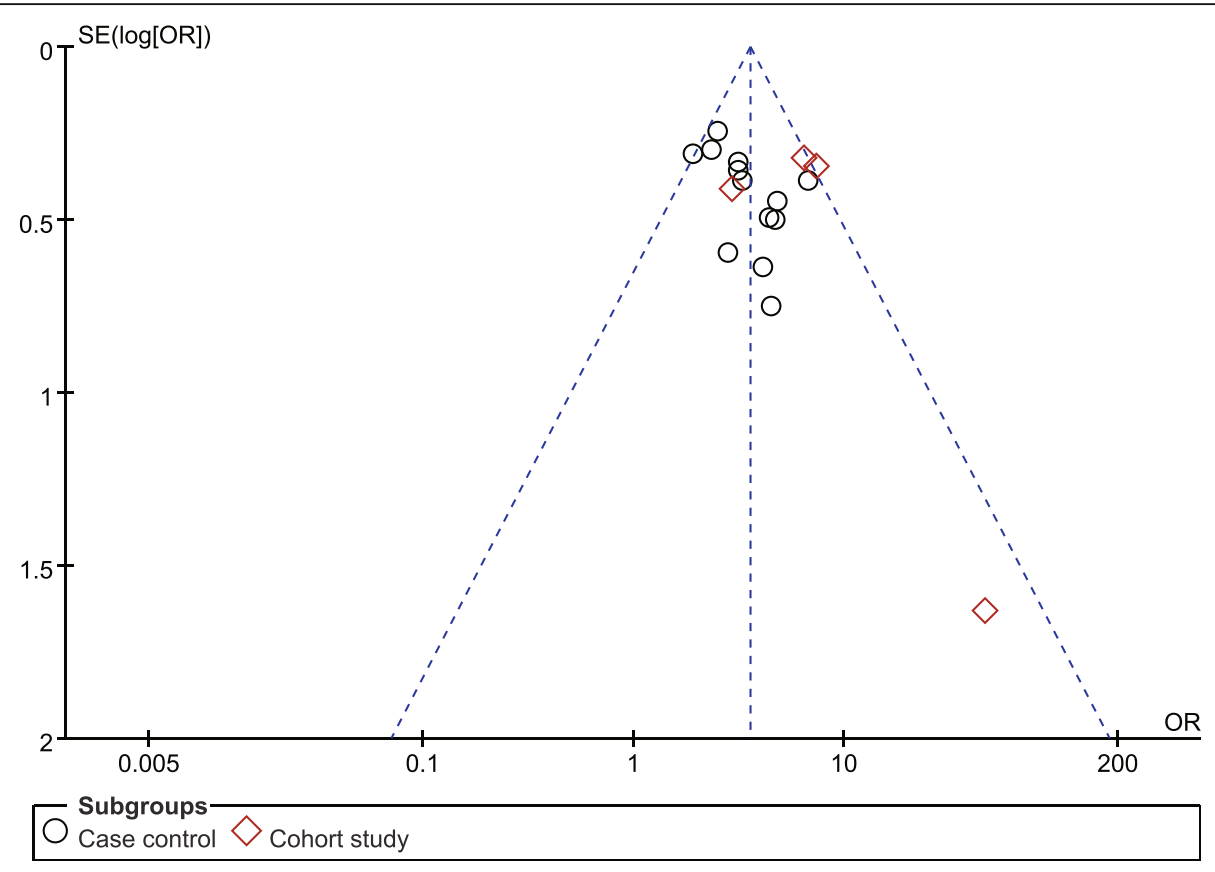

Fig. 12 Poor relationship between husband and wife funnel plot

parents-in-law is based on the environment rather than consanguinity. Sometimes they are reluctant to express their own feelings and opinions to their inlaws. New mothers feel very stressed when they have opinions different from their care providers. The situation may become even worse when conflicts occur with mothers-in-law. Because of the differences in backgrounds, values, identity, and logic of ideas, conflicts with respect to childcare between women and their mothers-in-law become prominent [18].

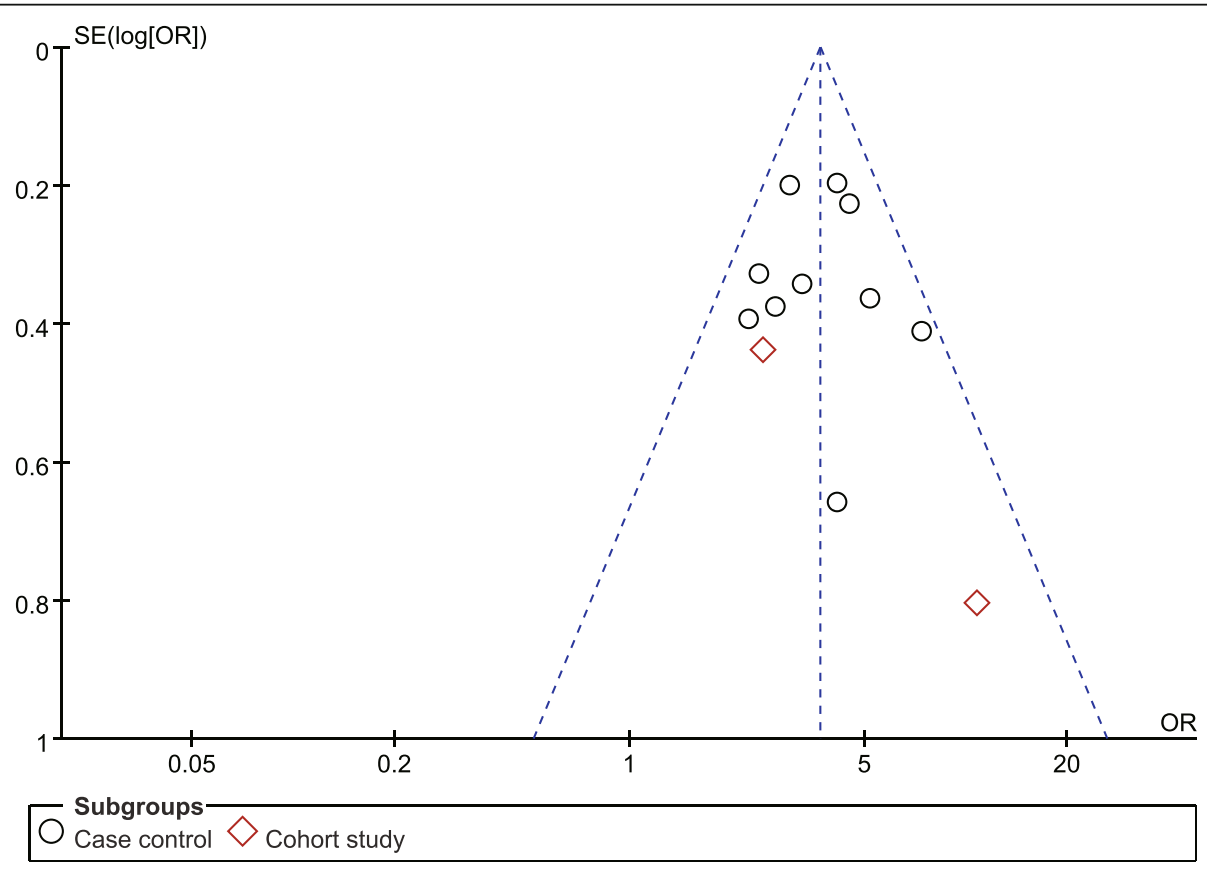

Fig. 13 Poor economic foundation funnel plot 


\section{Limitations}

This study had several inevitable limitations. First, some risk factors have received less attention; for example, non-uniform measurement standards and statistical difficulties have not been combined, such as the type of residence, postpartum wound recovery, postpartum work stress, and maternal occupation. Second, PPD is the result of the interaction of multiple factors, but due to methodological limitations, it is difficult to investigate the interaction among risk factors. Third, in terms of language selection, this study only includes literature in Chinese and English, which may lead to bias in the comprehensiveness of the literature search, thus affecting the research results and the intensity of the argument.

In addition, this study covers a wide range of research sites, including pregnant Chinese women in mainland China and Hong Kong, and Canadian immigrants. Although some Chinese individuals have emigrated to other countries, they have been influenced by traditional Chinese culture for a long time; thus their personality and their family structure are still similar to those of individuals in their motherland. In particular, many Chinese immigrant women continue to observe the Chinese traditional postpartum practices.

\section{Conclusion}

In conclusion, psychosocial risk factors for PPD mainly include prenatal depression, prenatal anxiety, a poor economic foundation, a poor relationship between husband and wife, a poor relationship between motherin-law and daughter-in-law, a lack of social support, unplanned pregnancy, the mother-in-law as the caregiver, and poor living conditions. These psychosocial risk factors are meaningful for identifying mothers "at-risk" during pregnancy even earlier. Meanwhile, some psychosocial interventions targeting these risk factors may be conducted during the pregnancy period to prevent PPD, such as interpersonal psychotherapy, mindfulness therapy, and psychoeducational programs.

\section{Abbreviations \\ PPD: Postpartum depression; EPDS: Edinburgh Postnatal Depression Scale; HADS: Hospital Anxiety and Depression Scale; SDS: Self-rating depression scale; SCL-90: Symptom checklist 90; BDI: Beck depression inventory; CES- D: Center for Epidemiological Studies Depression Scale; VIP: the China Science and Technology Journal Database; CNKI: China National Knowledge Infrastructure; CBM: China Biology Medicine Disc; RRs: Relative risks; ORs: Odds ratios; Cls: Corresponding 95\% confidence intervals; NOS: Newcastle-Ottawa Scale}

\section{Acknowledgements}

We appreciate the contribution of all people who participated in this study. We are also grateful for the valuable insight and feedback from the reviewers and the editorial team.

\section{Authors' contributions}

$W Q, F Z, J H, Y L$, and $Q L$ were all involved in the processes of study design, data extraction, and statistical analysis. WQ wrote the manuscript. JH and FZ were responsible for the selection of articles. All authors read and approved the final version of the manuscript.

\section{Funding}

This work was supported by Hebei Education Department Humanities and Social Sciences Young Talent Project (Grant numbers: BJ2016082) and National Natural Science Foundation of China (Grants 72074067).

The funding organization was not involved in the design of the study or collection, analysis, and interpretation of data, or in writing the manuscript.

\section{Availability of data and materials}

Data will be available from the corresponding author upon reasonable request.

\section{Declarations}

Ethics approval and consent to participate Not applicable.

\section{Consent for publication \\ Not Applicable.}

\section{Competing interests}

The authors declare that they have no conflict of interest.

Received: 24 August 2020 Accepted: 22 February 2021

Published online: 02 March 2021

\section{References}

1. O'Hara MW, McCabe JE. Postpartum depression: current status and future directions. Annu Rev Clin Psychol. 2013;9:379-407.

2. Diagnostic and Statistical Manual of Mental Disorders DSM-V. American Psychiatric Association. Washington, DC 2013;

3. Norhayati MN, Hazlina NH, Asrenee AR, Emilin WM. Magnitude and risk factors for postpartum symptoms: a literature review. J Affect Disord. 2015; 175:34-52.

4. Deng A, Xiong R, Jiang T, Luo Y, Chen W. Prevalence and risk factors of postpartum depression in a population-based sample of women in Tangxia community, Guangzhou. Asian Pac J Trop Med. 2014;7:244-9.

5. Mu T, Li Y, Pan H, Zhang L, Zha D, Zhang C, Xu R. Postpartum depressive mood (PDM) among Chinese women: a meta-analysis. Arch Women's Ment Health. 2019;22:279-87.

6. Johannsen BM, Larsen JT, Laursen TM, Ayre K, Howard LM, Meltzer-Brody S, Bech BH, Munk-Olsen T. Self-harm in women with postpartum mental disorders. Psychol Med. 2019;50:1-7.

7. Chang $\mathrm{H}$, Chen J, Huang $Y$, Chin-Hung CV. Prevalence and factors associated with depressive symptoms in mothers with infants or toddlers. Pediatr Neonatol. 2014;6:470-9.

8. Liu SX, Xia W. A Meta-analysis of risk factors of postpartum depression. Shanxi Med J. 2014;24:2847-9.

9. Beck CT. A Meta-analysis of predictors of postpartum depression. Nurs Res. 1996:45:297-303.

10. Klainin P, Arthur DG. Postpartum depression in Asian cultures: a literature review. Int J Nurs Stud. 2009:46:1355-73.

11. Guitian Z. A comparison of cultural characters between China and the west on "emotion and reason". Yin du Acad J. 1993:04:63-6.

12. Zhao SQ. Analysis of the Causes of the Transformation of Female Concepts in Modern China. J Shandong Normal Univ (Humanities and Social Sciences). 2015;04:34-43.

13. Wang D, Hong C. Chinese "openness" Western "Open" Personality Dimension and Chinese Personality. J Southwest University (Humanities and Social Sciences Edition). 2006;06:1-10.

14. Li M, Deng H. Study on correlation between personality characteristics and postpartum depression in primiparous women in Beijing city. Matern Child Health Care China. 2013:28:760-3.

15. Stang A. Critical evaluation of the Newcastle-Ottawa scale for the assessment of the quality of nonrandomized studies in meta-analyses. Eur J Epidemiol. 2010;25:603-5.

16. DerSimonian R, Laird N. Meta-analysis in clinical trials. Control Clin Trials. 1986;7:177-88. 
17. Joseph Lau JPAI. The case of the misleading funnel plot. BMJ. 2006;333:597600.

18. Siu BW, Leung SS, Ip P, Hung SF, O'Hara MW. Antenatal risk factors for postnatal depression: a prospective study of Chinese women at maternal and child health centres. BMC Psychiatry. 2012;12:22.

19. Zhao Y, Munro-Kramer ML, Shi S, Wang J, Zhu X. A longitudinal study of perinatal depression among Chinese high-risk pregnant women. Women Birth. 2018;31:395-402.

20. Pan XF, Lu ZM, Xiao J. Prospective study on occurrence of postnatal depression and its psychosocial risk factors. Matern Child Health Care China. 2004;11:28-30.

21. Zhao ZM, Zhao F, Yang X, Wen Y, Xu MY, Qi XR, Yang CX, Pan XF. Antenatal and postnatal depression in Chengdu, China: a longitudinal study. Chin J Dis Control Prev. 2018;22:1051-4.

22. Li HL, Li WL, Liu DH. Related facter of postpartum depressionin1994 cases in a hospital in Xi'an. Chin J Woman Child Health Res. 2017;10:1374-6.

23. Gu W, Tang YF, Huang YM, Shi SS. Study on incidence and risk factors associated with postpartum depression. Shanghai Med J. 2004;10:756-8.

24. Sun HL, Su H, Zhang J, Fan W. Analysis of Correlat ion factors of postpartum depression. J Kunming Med Univ. 2015;12:60-4.

25. Kang $A Q, W u C M$, Yang $L$. The incidence status and effect factors of postpartum depression in Rudong county. Chin J Fam Plan. 2015;10: 652-5.

26. Dennis CL, Brown HK, Wanigaratne S, Vigod SN, Grigoriadis S, Fung K, Marini F, Brennenstuhl S. Determinants of comorbid depression and anxiety postnatally: a longitudinal cohort study of Chinese-Canadian women. J Affect Disord. 2018:227:24-30.

27. Cai FY, Kuang L, Wang W, Li DQ, Cao J, Hui X. Prediction model for postpartum depression base on social psychological factors establishment and evaluation. Acad J Second Mil Univ. 2017;04:476-81.

28. $\mathrm{Hu} J$, Wang YQ. Study on related risk factors of patients with pre -and postnatal depression. Nurs Res China. 2010;09:765-7.

29. Gu SS, Qian Y, Chen H, Fu R, Wang FQ, Cheng JY. Status and related factors of postpartum depression in patients with second child. Chin J Woman Child Health Res. 2017:10:1177-80.

30. Zhang X, Li SF. Risk factors of postpartum depression of the parturients in Meilin and corresponding health care guidance. J Guangdong Med Univ. 2017;03:303-6.

31. Wu XQ, Ma J, Zhang Y, Zhu DL. Analysis of postpartum depression and its influencing factors in Futian District. Appl Prev Med. 2019;02:95-8.

32. Shen R. Investigation and analysis of postpartum depression status and its influence factors and psychological intervention research. Master Thesis, Shanxi Medical University; 2011

33. Yang $L P, X u Q, Q i$ L. Risk factors and preventive measures of postpartum depression. Chin Clin Nurs. 2016;06:501-4.

34. Zhang $X$, Tu Q, Xi W, Jiang YL, Gao Y. A study of incidence and the related factors of postpartum depression. Chin J Psychiatry. 2001;04:47-9.

35. Yin J. The risk factors of postpartum depression and nursing strategy. Hebei Med. 2011;12:1673-6.

36. Huang $\mathrm{GH}$. Analysis of pathogenic factors of postpartum depression. Today Nurse. 2012;06:29-30.

37. Song LQ. Discussion on related factors and nursing countermeasures of postpartum depression. Today Nurse. 2012;01:77-9.

38. Wang XL, Dong Y, Zhou XM, Hou GZ, Qi JL, Guo L, Wang J. Risk factors of postnatal depression among women in urban nuclear family. China J Health Psychol. 2013;04:513-5.

39. Lin YP, Gu SQ, Shen HL. Analysis of the incidence and influencing factors of postpartum depression in 2023 parturients. Chin Rural Health Serv Adm. 2014:08:979-80

40. Zhou Q, Zhang FZ. Analysis on related factors of postpartum depression and its preventive measures. Med Recapitulate. 2014;18:3417-8.

41. Wang YC, Zou T, Deng B. Cross-sectional survey of postpartum depression in the old town of Guiyang City and analysis of its influencing factors. Pract Prev Med. 2014;10:1257-60

42. Chen XD, Jiang WW. Influencing factors and intervention measures of postpartum depression in rural pregnant women. Chin J Public Health Manage. 2017:06:877-9.

43. Yan J, Qin SH, Zhao YM, Li XY, Kun ML. Analysis of the incidence and influencing factors of postpartum depression in a grass-roots hospital. Guide China Med. 2018;10:135-6.
44. Han YH. Analysis of influencing factors of postpartum depression of pregnant women and related nursing countermeasures. J Shanxi Med Coll Contin Educ. 2018;01:111-3.

45. He KL, Tao M, Zhu XZ. Analysis of related factors of postpartum depression and preventive measure. Chin J Woman Child Health Res. 2019;06:705-7.

46. Yu J. The study of postpartum depression in Shanghai. Master Thesis, Fudan University; 2010

47. Zhang Y, Zou S, Cao Y, Zhang Y. Relationship between domestic violence and postnatal depression among pregnant Chinese women. Int J Gynecol Obstet. 2012;116:26-30.

48. Zhang H, Zhou P, Liu L. Analysis of relevant factors of postpartum depression in rural primiparas. Med J Chin People's Health. 2014;4:1-5.

49. Li J, Wang X. Analysis of influencing factors of postpartum depression in parturients in northern Anhui. J Fuyang Inst Technol. 2104;02:51-53.

50. Liu S, Hu Y. Postpartum depression status and the influencing factors analysis. Chin Clin Nurs. 2015;04:281-3.

51. Liu Y, Li X, Zhang H, Liu Y. Current situation of postpartum depression and analysis of related social and psychological factors. Matern Child Health Care China. 2015;22:3794-6.

52. Chen L, Ding L, Qi M, Jiang C, Mao X, Cai W. Incidence of and socialdemographic and obstetric factors associated with postpartum depression: differences among ethnic Han and Kazak women of northwestern China. Peerj. 2018;6:e4335.

53. Wang P. Analysis of related factors in 168 patients with postpartum depression. J Shandong Med Coll. 2013;04:278-80.

54. Li Y. Related risk factors for postpartum depression and its nursing intervention. J Clin Med Pract. 2019;6:126-8 132

55. Guan A. Postpartum Depression Status and Influencing Factors of Hospitalized Parturients in Three General Hospitals of Baotou. Master Thesis, Shandong University; 2012

56. Zhang Y. Study of psychosocial factors for postpartum depression among 479 Wome. Doctor Thesis, Huazhong University of Science and Technology; 2011

57. Aiwen D, Ribo X, Jiang T, Luo Y, Wei D. Epidemiological study of postpartum depression among migrant women at Tianhe District of Guangzhou. J Pract Med. 2014;10:1648-51.

58. Han YH. Investigation on the incidence and influencing factors of postpartum depression in a certain area. Guide China Med. 2015;12: 107-8.

59. Liu $\mathrm{H}$. Analysis on the influencing factors of postpartum depression in rural women in Doumen District of Zhuhai city and the prevention countermeasures. Matern Child Health Care China. 2017;14:3100-2.

60. Zhou H, Qin Z, Yang X, Tang J, Mo J, Qian Q. Investigation and analysis of related factors of postpartum depression among women in Changzhou. Matern Child Health Care China. 2019:6:1347-51.

61. Liu $P, Y a X$. Analysis of influencing factors and nursing strategies of postpartum depression. Matern Child Health Care China. 2014;33:5402-3.

62. Xie R, He G, Koszycki D, Walker M, Wen SW. Fetal sex, social support, and postpartum depression. Can J Psychiatry. 2009:54:750-6.

63. Pan $L$. An analysis of the higher risk factors engendering postpartum depression. Health Res. 2015;05:544-5.

64. Yang W. Discussion on risk factors of postpartum depression in rural women and nursing intervention. Med Forum. 2020;32:4718-20.

65. Yun-xia $H$, Jiang-ye K. Investigation on the occurrence of depression in 784 postpartum women. China Foreign Med Treat. 2020:39:82-4.

66. Li Q, Yang S, Xie M, Wu X, Huang L, Ruan W, Liu Y. Impact of some social and clinical factors on the development of postpartum depression in Chinese women. BMC Pregnancy Childb. 2020;20:226.

67. Dan W, Jun-xia Z, Zhen-yun M, Hong-xia Z, Xiao-dong Z, Xue-yi W, Ping G. Discussing on the research of heterogeneity in Meta-analysis. Chin J Evid Based Med. 2009;9:1115-8.

68. Palumbo G, Mirabella F, Gigantesco A. Positive screening and risk factors for postpartum depression. Eur Psychiat. 2017:42:77-85.

69. Lee DT, Yip AS, Leung TY, Chung TK. Ethnoepidemiology of postnatal depression. Prospective multivariate study of sociocultural risk factors in a Chinese population in Hong Kong. Br J Psychiatry. 2004;184:34-40.

70. Chen W, Li Y. Analysis of the effect of prenatal anxiety and depression on obstetric complications and pregnancy outcomes. Modern Medical Imageology. 2018;08:2886-7.

71. Oztora S, Arslan A, Caylan A, Dagdeviren HN. Postpartum depression and affecting factors in primary care. Niger J Clin Pract. 2019;22:85-91. 
72. Xiong R, Deng A, Wan B, Liu Y. Prevalence and factors associated with postpartum depression in women from single-child families. Int J Gynecol Obstet. 2018;141:194-9.

73. Zhang YL, Zhang QG, Han JL, et al. Med Philos (Clinical Decision Making Forum Edition). 2008;02:57-8.

74. Malus A, Szyluk J, Galinska-Skok B, Konarzewska B. Incidence of postpartum depression and couple relationship quality. Psychiatr Pol. 2016;50:1135-46.

75. Fu D, Qin Y. An analysis of Chinese and Western family culture from the perspective of comparative culture. J Taiyuan Normal Univ (Social Science Edition). 2017:2:29-31.

76. Deng W. On the cultural differences between China and the west from the comparison of family education between China and the United States. J Ansh Un Univers Ity. 2009;11:23-5.

77. Steinberg S. Childbearing research: a transcultural review. Soc Sci Med. 1996;43:1765-84.

78. Leung SK, Arthur D, Martinson IM. Perceived stress and support of the Chinese postpartum ritual "doing the month". Health Care Women Int 2005;26:212-24.

\section{Publisher's Note}

Springer Nature remains neutral with regard to jurisdictional claims in published maps and institutional affiliations.

- fast, convenient online submission

- thorough peer review by experienced researchers in your field

- rapid publication on acceptance

- support for research data, including large and complex data types

- gold Open Access which fosters wider collaboration and increased citations

- maximum visibility for your research: over $100 \mathrm{M}$ website views per year

At $\mathrm{BMC}$, research is always in progress. 